K.A. van der Hucht, G. Koenigsberger \& P.R.J. Eenens, eds.

\title{
The effect of micro-turbulence in O-type star analyses
}

\author{
M.R. (Charo) Villamariz and Artemio Herrero \\ Instituto de Astrofísica de Canarias, 38200 La Laguna, Tenerife, Spain
}

\begin{abstract}
We study here the influence of micro-turbulence in the synthesis of $\mathrm{H}$ and $\mathrm{He}$ lines, in order to quantify its effect on the determination of stellar parameters of O-type stars, in particular on the stellar $\mathrm{He}$ abundance. We find that only He I lines and He II $\lambda 4686$ are considerably affected, the effect being more relevant for models corresponding to supergiant stars. We also find that neglecting micro-turbulence in the analyses of O-type stars introduces deviations in the derived parameters that are within our standard error box. The He content is not or slightly reduced, thus micro-turbulence cannot be responsible for the He-discrepancy to all its extent.
\end{abstract}

\section{Introduction}

A considerable number of $\mathrm{O}$ - and early B-type stars in the sample of Herrero et al. (1992) (hereafter Paper I) were found to be He-enriched, when comparing their spectroscopic He-abundances with the ones predicted by standard stellar evolutionary models (the so-called He-discrepancy). There has been a lot of work since then to try and explain this. One of the possible explanations, supported by the results of McErlean et al. (1998), is that in Paper I micro-turbulence was not taken into account when performing the analyses. However, the former results only refered to early B- and late O-type supergiants, while the Herrero et al. sample covered stars from $\mathrm{O} 5$ to B0.5 of all luminosity class. Thus we decide to study the behaviour with micro-turbulence of $\mathrm{H}$ and $\mathrm{He}$ model profiles, the ones used to analyse these spectra, in the parameter range typical from $\mathrm{O}$ to early B stars, in order to see how they are affected by it and how the parameters obtained from the analyses can change. Finally, we perform new analyses for four stars, with a fixed value for the micro-turbulence of $15 \mathrm{~km} \mathrm{~s}^{-1}$, to quantify these changes, specially in the He-abundance: $15 \mathrm{~km} \mathrm{~s}^{-1}$ is a suitable value in early stars.

\section{Micro-turbulence in $\mathrm{H}-$ and He-profiles}

We calculate profiles in NLTE planeparallel hydrostatic models with parameters $T_{\text {eff }}=30000-45000 \mathrm{~K}, \log g=3.0-4.0$, and $\epsilon=0.08-0.25$. We let microturbulence change between 0 and $20 \mathrm{~km} \mathrm{~s}^{-1}$. The codes used are ALI (Kunze 1995) and DETAIL \& SURFACE (Butler \& Giddings 1985). We have included lineblocking extensively in the present work; for details see Herrero et al. (1999). Micro-turbulence is introduced in DETAIL \& SURFACE by adding an extra Doppler-width to the thermal broadening. $\mathrm{H} \gamma, \mathrm{H} \beta, \mathrm{He}$ I 4387, 4471, 4922, and $\mathrm{He}$ II $4199,4541,4686 \AA$ were the lines used for the analyses in Paper I. Of all these, only $\mathrm{He}$ I lines and the core of He II $\lambda 4686$ are affected by micro-turbulence, 
showing the corresponding behaviour of saturated and non-saturated lines, in each case. For the rest of the lines Stark-broadening dominates the profiles and hides the effect of micro-turbulence. Effects are more relevant as $\log g$ decreases, thus supergiants will show the larger changes in the parameters.

\section{Analysis with micro-turbulence}

Values of micro-turbulence of 10 to $12 \mathrm{~km} \mathrm{~s}^{-1}$ are found for late O-type and early B-type stars ( Gies \& Lambert 1992), and as we have seen, supergiant spectra are the most sensitive to micro-turbulence, so we decide to analyse two late and two early O-type supergiants with a value for the micro-turbulence of $15 \mathrm{~km} \mathrm{~s}^{-1}$, in order to quantify the changes in their parameters.

Table 1. Analyses with and without micro-turbulence. Parameters are determined with an accuracy of $1000 \mathrm{~K}$ in $T_{\text {eff }}, 0.1$ in $\log g$ and 0.03 in $\epsilon$, for a fixed $v_{\text {turb }}$.

\begin{tabular}{llccccc}
\hline star & $\begin{array}{l}\text { spectral } \\
\text { type }\end{array}$ & $\begin{array}{c}v_{\mathrm{r}} \sin i \\
\left(\mathrm{~km} \mathrm{~s}^{-1}\right)\end{array}$ & $\begin{array}{c}T_{\mathrm{eff}} \\
(\mathrm{kK})\end{array}$ & $\begin{array}{c}\log g \\
(\mathrm{cgs})\end{array}$ & $\begin{array}{c}\epsilon \\
\frac{N(\mathrm{He})}{N(\mathrm{He})+N(\mathrm{H})}\end{array}$ & $\begin{array}{c}v_{\text {turb }} \\
\left(\mathrm{km} \mathrm{s}^{-1}\right)\end{array}$ \\
\hline \multirow{2}{*}{ HD 14947 } & O5 If & \multirow{2}{*}{140} & 45.0 & 3.50 & 0.15 & 0 \\
& & & 45.0 & 3.45 & 0.15 & 15 \\
HD 5689 & O6 & \multirow{2}{*}{250} & 40.0 & 3.40 & 0.25 & 0 \\
& & & 40.0 & 3.35 & 0.25 & 15 \\
HD 18409 & O9.7 Ib & \multirow{2}{*}{160} & 31.5 & 3.10 & 0.11 & 0 \\
& & & 32.0 & 3.10 & 0.09 & 15 \\
HD 210809 & \multirow{2}{*}{ O9 Iab } & \multirow{2}{*}{120} & 33.5 & 3.10 & 0.10 & 0 \\
& & & 34.5 & 3.15 & 0.08 & 15 \\
& & & & & & \\
\hline
\end{tabular}

\section{Conclusions}

Only He I model lines and He II $\lambda 4686 \AA$ are considerably affected by the presence of micro-turbulence, thus the models for supergiants are the most sensitive. Our analyses, with a value of the micro-turbulence of $15 \mathrm{~km} \mathrm{~s}^{-1}$ introduce changes in the parameters obtained, but not beyond our standard error box. The Heabundance is lowered by 0.02 in the later type supergiants, earlier types keep the same value. So micro-turbulence cannot explain the He-discrepancy, neither for earlier type stars, nor for the very overabundant stars.

\section{References}

Butler, K., Giddings, J. 1985, in: Newsletter on Analysis of Astron. Spectra, 9, Daresbury Laboratory

Gies, D.R., Lambert, D.L. 1992, ApJ 387, 673

Herrero, A., Kudritzki, R.P., Vílchez, J.M. et al. 1992, A\&A 261, 209

Herrero, A., Villamariz, M.R., Puls, J. 1999, in preparation

Kunze, D. 1995, PhD Thesis, Ludwig-Maximillian University, Munich

McErlean, N.D., Lennon, D.J., Dufton, P.L. 1998, A\&A 329, 613 